\title{
GsMATE encoding a multidrug and toxic compound extrusion transporter enhances aluminum tolerance in Arabidopsis thaliana

Qibin Ma ${ }^{1,2,3 \dagger}$, Rong $\mathrm{Yi}^{1,2,3 \dagger}$, Lu Li ${ }^{1,2,3}$, Zhongyi Liang ${ }^{1,2,3}$, Tingting Zeng ${ }^{1,2,3}$, Yu Zhang ${ }^{1,2,3}$, He Huang ${ }^{4}$, Xiao Zhang ${ }^{1,2,3}$, Xiangli Yin ${ }^{1,2,3}$, Zhandong Cai ${ }^{1,2,3}$, Yinghui Mu ${ }^{1,2,3}$, Yanbo Cheng ${ }^{1,2,3}$, Qiaoying Zeng ${ }^{1,2,5}$, Xiuping $\mathrm{Li}^{1,2,6^{*}}$ and Hai Nian ${ }^{1,2,3^{*}}$

\begin{abstract}
Background: Multidrug and toxic compound extrusion (MATE) transporters, which exist widely in plants, function as crucial regulators in plant resistance to aluminum (Al) toxicity by inducing citrate efflux. However, the functions of most MATE family members in soybean (Glycine soja) remain to be elucidated.

Results: Expression pattern analysis showed that GSMATE was constitutively expressed in different soybean organs, with the highest level in root compared with those in stem, leaf and cotyledon. In addition, Al stress induced expression of GSMATE in soybean. Temporal analysis indicated that GSMATE expression was greatly enhanced by increasing concentrations of aluminum $\left[\mathrm{Al}^{3+}\right]$ after short exposure, reaching the high levels detected in the BW69 (Al-resistant) and the JW81 (Al-sensitive) lines of Glycine soja of wild soybean at $6 \mathrm{~h}$ and $8 \mathrm{~h}$, respectively. Furthermore, transient GSMATE expression in Arabidopsis protoplasts showed that GsMATE protein localized to the plasma membrane. Overexpression of GSMATE on an Arabidopsis columbia-0 (Col-0) background resulted in increased Al tolerance in transgenic plants. Analysis of hematoxylin staining showed that the roots of GSMATE transgenic lines were stained less intensely than those of the wild-type exposured to the same $\mathrm{AlCl}_{3}$ concentrations. Therefore, GSMATE enhanced the resistance of transgenic plants to Al toxicity by reducing Al accumulation in Arabidopsis roots.
\end{abstract}

Conclusions: In summary, our results indicate that GSMATE is responsive to aluminum stress and may participate in the regulation of sensitivity to $\mathrm{Al}$ toxicity in Arabidopsis. In addition, the GSMATE protein is an Al-induced citrate transporter of the MATE family and exerts an essential role in Al tolerance in Glycine soja.

Keywords: GSMATE, Al tolerance, Glycine soja, Arabidopsis thaliana

\section{Background}

Acid soils ( $\mathrm{pH}<5.5$ ), representing up to $50 \%$ of arable land, are widely distributed in developing countries in Africa, Asia, and South America [1-3]. Solubilized aluminum (Al), the most toxic trivalent cation $\left(\mathrm{Al}^{3+}\right)$ which forms an $\mathrm{Al}$ complex in aluminosilicate clays ( $\mathrm{pH}<5.5)$, is a major limiting factor for plant growth and crop yield in acidic soils [4]. $\mathrm{Al}^{3+}$ toxicity primarily damages the root apex, causing significant reductions in plant growth and development by

\footnotetext{
* Correspondence: 382063902@qq.com; hnian@scau.edu.cn

${ }^{\dagger}$ Qibin Ma and Rong Yi contributed equally to this work.

${ }^{1}$ The State Key Laboratory for Conservation and Utilization of Subtropical

Agro- bioresources, South China Agricultural University, Guangzhou,

Guangdong 510642, People's Republic of China

Full list of author information is available at the end of the article
}

affecting the plasma membrane structure, inducing root cell death and inhibiting nutrient uptake $[5,6]$. To date, two main types of Al-resistance mechanisms have been investigated in most plant species such as maize, wheat, and sorghum. The exclusion mechanism prevents $\mathrm{Al}$ from entering the root apex, while tolerance mechanisms detoxify and sequester $\mathrm{Al}$ in plants $[1,3]$. Under $\mathrm{Al}$ stress, plants enhance their resistance to $\mathrm{Al}$ toxicity by root exudation of organic acids including malate, citrate and oxalate [7-11]. Multidrug and toxic compound extrusion (MATE) transporters have recently become the most categorized multidrug efflux transporter family. These proteins couple with substrate translocation across the plasma membrane with an electrochemical gradient of cations (such as $\mathrm{H}^{+}$or $\mathrm{Na}^{+}$

(C) The Author(s). 2018 Open Access This article is distributed under the terms of the Creative Commons Attribution 4.0 International License (http://creativecommons.org/licenses/by/4.0/), which permits unrestricted use, distribution, and 
ions) [12]. X-ray crystallography indicated a unique structural topology of the predicted 12 transmembrane (TM) helices in the MATE transporter that was distinct from that of other multidrug resistance transporters [13]. MATE transporters exist widely in bacteria, fungi, mammals and plants [14]. Many MATE genes, which encode proteins that induce citrate efflux in response to aluminum toxicity, have also been investigated and characterized in plants [1]. Using map-based cloning, SbMATE and HvAACT1 were the first MATE transporters shown to be involved in detoxification of $\mathrm{Al}$ in sorghum and barley $[15,16]$. Since then, a number of MATE genes have been reported to be involved in the $\mathrm{Al}$-induced secretion of citrate in herbaceous plants. These genes include AtMATE in Arabidopsis [6, 17], TaMATE in wheat [18], ZmMATE1 in maize [19], ScFRDL1 in rye [20], HvAACT1 in wheat and barley [21], SbMATE in barley [22], BoMATE in Arabidopsis [23], OsFRDL2 in rice [24], FeMATE1 and FeMATE2 in buckwheat [17], BdMATE in Setaria viridis [25], and MtMATE66 in Medicago truncatula [26]. All these homologous genes encode MATE proteins that are required for external Al-resistance, and are primarily localized to the root epidermis cells [16, 17, 20, 23]. Some plant MATE transporters play diverse roles in iron homeostasis [26-32]; heavy metal [33], and toxin resistance [34]; vacuolar transport of nicotine [35-37]; chloride channels [38]; abscisic acid (ABA) efflux [39]; transport of secondary metabolites such as alkaloids, flavonoids, and anthocyanins [36, 39-42]; hypocotyl cell elongation [43]; export of hydroxycinnamic acid amides [44]; organ initiation [45]; regulation of lateral organ size and initiation rate [46]; plant growth and development [47]; the establishment of plant disease resistance [48]; and resistance to viruses [49], etc.

Previous studies have shown that the MATE proteins are a large family of multidrug efflux transporters in plants [12]. Many putative MATE transporters have been predicted and identified by genome-wide analysis and/or other methods in plants. These putative MATE transporters include 45 in Oryza sativa [50], 49 in maize [51], 56 in Arabidopsis thaliana [33, 50], 67 in tomato [7], 70 in Medicago truncatula [26, 52], 71 in Populus [1], 70 MATE genes in Gossypium raimondii and 68 MATE genes in Gossypium arboretum [53], and 117 in Glycine max [54]. Compared with other plant species, soybean has the largest MATE family with 117 putative MATE transporters predicted by genome-wide association analysis and RNA-seq Atlas of Glycine $\max$ [54]. However, few studies have been conducted on MATE transporters in soybean. To date, only two MATE transporters, GmFRD3a and GmFRD3b, have been reported to play a role in iron homeostasis in soybean $[31,55]$. In the current study, GSMATE (accession number: BM732932.1) was cloned from the wild soybean root of the BW69 line (Al-resistant) of Glycine Soja, and the gene expression pattern was detected in response to $\mathrm{Al}$ treatment. The function of GsMATE was identified and characterized on subcellular localization and citrate transport activity along with phenotypic analysis of transgenic overexpression lines of Arabidopsis. We hypothesized that GsMATE would enhance Al tolerance in Arabidopsis via Al-induced secretion of citrate from the root.

\section{Methods}

\section{Plant materials and growth conditions}

The BW69 (Al-resistant) and the JW81 (Al-sensitive) lines of Glycine soja were used to clone GSMATE for investigation of GSMATE expression patterns in response to $\mathrm{Al}$ stress. The wild soybean seed coat was lacerated gently with a single-sided blade on the back of the hilum. Then, all seeds of wild soybean were grown under growth chamber $\left(22-25{ }^{\circ} \mathrm{C}, 12-\mathrm{h} / 12-\mathrm{h}\right.$ light/dark cycle) as described previously by Zeng [56]. After germination, the seedlings were pre-cultured for $48 \mathrm{~h}$ in surface-sterilized vermiculite and transplanted into the nutrient solution ( $\mathrm{pH} 5.8$ ) after the spread cotyledons. After two days cultured at $22 / 25^{\circ} \mathrm{C}$ with a 12-h/12-h light/dark cycle, the soybean seedlings were treated in aluminum solutions [56].

Ecotype Col-0 of Arabidopsis was used for GSMATE gene transformation. All the surface-sterilized Arabidopsis seeds (wild-type and transgenic lines) were cultivated on 1/2 Murashige and Skoog (MS) agar medium in darkness for 2 days at $4{ }^{\circ} \mathrm{C}$. The Arabidopsis seedlings were then transferred to new $1 / 2 \mathrm{MS}$ medium containing aluminum concentration gradients for continuous culture for several days at $22{ }^{\circ} \mathrm{C}$ under long-day conditions until all the samples were taken [57].

\section{GsMATE expression analysis}

To analyze the tissue expression pattern of GSMATE, the BW69 and JW81 lines were germinated in sterilized vermiculite. The seedlings with open cotyledons were cultured in the nutrient solutions for four days. Samples of root, stem, leaf and cotyledon were taken from the seedlings ( $n=10$ plants per group), frozen in liquid nitrogen, and then stored at $-80{ }^{\circ} \mathrm{C}$ [56]. To analyze the influence of the $\mathrm{Al}$ concentration gradient on gene expression, the BW69 and JW81 lines were planted and germinated using the method described previously [56]. In brief, after pre-incubation for two days, the soybean seedlings were transferred and cultured in the solutions of $0,25,50,75$, and $100 \mu \mathrm{M} \mathrm{AlCl}{ }_{3}(\mathrm{pH} 4.3,0.5 \mathrm{mM}$ $\mathrm{CaCl}_{2}$ ), respectively; three replicates of 20 seedlings were prepared for each group. After treatment with aluminum solutions for $6 \mathrm{~h}$, root tip samples $(6 \mathrm{~cm}$ long) were obtained from the seedlings ( $n=10$ plants used per group). Samples were frozen in liquid nitrogen and then stored at $-80{ }^{\circ} \mathrm{C}$ [56]. To analyze the pattern of GSMATE expression in different root segments in response to $\mathrm{Al}$ 
toxicity, the soybean seedlings were prepared using the method described previously. Samples were obtained from seedling roots (sections $0-2,2-4$, and $>4 \mathrm{~cm}$ ) after $6 \mathrm{~h}$ of treatment in $\mathrm{Al}$ solutions $(n=10$ plants per group), frozen in liquid nitrogen and stored at $-80{ }^{\circ} \mathrm{C}$ for further analysis [56]. To analyze the temporal expression pattern of GsMATE in response to Al toxicity, two-day-old seedlings were cultured in a solution of $0.5 \mathrm{mM} \mathrm{CaCl} 2(\mathrm{pH} 4.3)$ for $12 \mathrm{~h}$. Root tip samples $(6 \mathrm{~cm}$ long) were obtained from the seedlings after the time course treatments set as 0,6 and $12 \mathrm{~h}$, respectively. The other seedlings were then transferred to a solution of $50 \mu \mathrm{M} \mathrm{AlCl}$ ( $\mathrm{pH} \mathrm{4.3,0.5} \mathrm{mM} \mathrm{CaCl}$ ) and cultured for $24 \mathrm{~h}$ ( $n=20$ seedlings per group). Root tip samples (6 $\mathrm{cm}$ long) were obtained from the seedlings treated after 2, 4, 6, 8, 12, and $24 \mathrm{~h}$, respectively. All the samples were frozen in liquid nitrogen, and stored at $-80{ }^{\circ} \mathrm{C}$ for further analysis [56].

\section{Cloning of GsMATE}

According to our previous analysis of the expression profiles of genes involved in acidic aluminum tolerance in BW69 and JW81 lines, one Al-induced GsMATE gene was identified by screening the database of the National Center for Biotechnology Information (NCBI) with the sequence information (unpublished data); the gene was assigned the accession number of BM732932.1. Specific primers (Additional file 1: Table S1) were designed to amplify the full-length sequence of GSMATE by RT-PCR using Super-Fidelity DNA polymerase (Phanta Max, Vazyme Biotech Co., Ltd.; Nanjing, China). Total RNA was extracted from the seedlings of BW69 under the treatment of $25 \mu \mathrm{M} \mathrm{AlCl}_{3}$ (pH 4.3, $0.5 \mathrm{mM} \mathrm{CaCl}_{2}$ ) using TRIzol reagent (Invitrogen). The cDNA was generated using $2 \mu \mathrm{g}$ of total RNA by reverse transcription with SuperScript II reverse transcriptase (Invitrogen). Using cDNA of BW69 as a template, the reaction of RT-PCR was performed in a total volume of $20 \mu \mathrm{l}$ according to a previously described method [57] followed by the amplification program: DNA denaturation at $94{ }^{\circ} \mathrm{C}$ for $30 \mathrm{~s}$, annealing at $72{ }^{\circ} \mathrm{C}$ for $30 \mathrm{~s}$, elongation at $72{ }^{\circ} \mathrm{C}$ for $1 \mathrm{~min}$ and $30 \mathrm{~s}$ (35 cycles). The GsMATE PCR product was isolated by $1 \%$ agarose gel electrophoresis (GenStar Kit, Genstar Development Company, Canada) and then inserted into the multiple cloning site of the $\mathrm{pLB}$ vector (Tiangen Rapid DNA Ligation Kit, Beijing, China). Clones of $E$. coli that were positively transformed into competent cells of DH5 $\alpha$ strains with the GsMATE-pLB vector using the method of heat-shock were identified by PCR, enzyme digestion and sequencing (Sangon Biotech (Shanghai) Co., Ltd., China) to obtain the full cDNA sequence of GsMATE. The methods of PCR identification and enzyme digestion for the positive clones were previously described in detail $[57,58]$.

\section{Sequence analysis}

Multiple alignments of sequences were carried out, and a homology tree was generated using DNAMAN software. The nucleotide and amino acid sequences were used to search for GsMATE and/or its homologous proteins using the BLAST network servers of the NCBI (https://blast.ncbi.nlm.nih.gov/Blast.cgi) and phytozome (https://phytozome.jgi.doe.gov/pz/portal.html) [57]. Prediction of the transmembrane topology of GsMATE protein was performed using the TMHMM Server website v. 2.0: http://www.cbs.dtu.dk/services/TMHMM/.

\section{Plasmid construction and transformation of GsMATE in Arabidopsis}

The 1,503 bp GsMATE coding sequence (CDS) (Additional file 2) amplified from the GsMATE-pLB vector was inserted into the BamHI and KpnI sites of a pCAMBIA1301 vector with a $\beta$-glucuronidase (GUS) reporter to generate the pCAMBIA1301-GsMATE fusion construct under the control of the cauliflower mosaic virus $35 \mathrm{~S}$ (CaMV 35S) promoter using specific primers (Additional file 1: Table S1). Agrobacterium tumefaciens GV3101 was then transformed with the pCAMBIA1301-GsMATE vector by electroporation, and full-flowering Arabidopsis plants were transformed using the floral dip method described previously [59]. All the GsMATE transgenic plants were obtained by hygromycin screening using 1/2 MS media.

\section{Localization of the GsMATE-GFP fusion protein}

Localization of the GsMATE protein was performed using a previously described method [57]. Specific primers were used to amplify the full GSMATE CDS (Additional file 1: Table S1). The PCR product of the GsMATE CDS was subcloned into the BamHI and KpnI sites of the pYL322-d1 vector to generate a GsMATE-GFP fusion construct under the control of CaMV $35 \mathrm{~S}$ promoter. The pYL322-d1-GsMATE-GFP construct was confirmed by sequencing and used for transient transformation of Arabidopsis protoplasts by heat-shock. Transformed Arabidopsis protoplasts were then observed under a confocal laser scanning microscope (Leica) to characterize GsMATE protein expression [57, 60].

\section{Quantitative real-time PCR}

Total RNA was extracted from the seedlings of soybean or Arabidopsis using TRIzol reagent (Invitrogen) and treated with RNase-free DNase (Promega). Total RNA of $2 \mu \mathrm{g}$ was used to generate cDNA by reverse transcription with SuperScript II reverse transcriptase (Invitrogen). The cDNA from each sample was then diluted to 4 and $8 \mathrm{ng} / \mathrm{ml}$. Triplicate quantitative assays were performed on $1 \mathrm{ml}$ of each cDNA dilution with the SYBR Green Master mix and the SsoFast EvaGreen Supermix Kit (BIO-RAD) on an ABI 7900 sequence detection system according to the 
manufacturers' instructions. The data were normalized using the reference gene $\beta$-tubulin. The quantitative variation between the examined replicates was evaluated by the $2^{-\Delta \Delta \mathrm{Ct}}$ method [57]. Details of the GsMATE and $\beta$-tubulin specific primers were listed in (Additional file 1: Table S1).

\section{Acidic aluminum treatment in transgenic Arabidopsis} For short-term $\mathrm{AlCl}_{3}$ treatment, $\mathrm{T}_{3}$ generation Arabidopsis seeds were sown in plastic petri dishes $(10 \mathrm{~cm}$ diameter) filled with $1 / 2$ MS phytagel medium ( $\mathrm{pH} 5.8$ ) and incubated at $4{ }^{\circ} \mathrm{C}$ in the dark for four days before transferred to a culture room (16-h light/8-h dark) at $22{ }^{\circ} \mathrm{C}$ for four days. Then, Arabidopsis seedlings were transferred to agarose medium containing $\mathrm{AlCl}_{3}$ (pH 4.5, $0.5 \mathrm{mM} \mathrm{CaCl} 2)$ and cultured at $22{ }^{\circ} \mathrm{C}(16-\mathrm{h} / 8$-h light/ dark) for another two days. The seedlings were then exposed to $\mathrm{AlCl}_{3}(\mathrm{pH} 4.5,0.5 \mathrm{mM} \mathrm{CaCl}$ ) concentration gradients $(0,50,100$ and $200 \mu \mathrm{M})$, and main root measurements were carried out using a previously described method [61]. For long-term $\mathrm{AlCl}_{3}$ treatment, the Arabidopsis seedlings were cultured for 7 days or more to observe the Al-resistance phenotypes and/or measure the main root length [62].

Hematoxylin staining was used to further investigate the resistance of transgenic lines of Arabidopsis to $\mathrm{Al}$ toxicity [11]. Arabidopsis seedlings were prepared according to the previously described method. The seedlings were then transferred into $\mathrm{AlCl}_{3}$ solutions $(\mathrm{pH} 4.5$, $0.5 \mathrm{mM} \mathrm{CaCl}_{2}$ ) for $6 \mathrm{~h}$. After 30 mins rinse in ultrapure water, the roots of the Arabidopsis seedlings were stained with hematoxylin for $30 \mathrm{~min}$. After another 30 mins rinse in ultrapure water, the Arabidopsis phenotypes were recorded [11].

\section{Statistical analysis}

All data were represented as the mean \pm SD of three biological replicates. The t-test at $p=0.05$ was performed to identify significant differences between observation values using SPSS20 software [57].

\section{Results}

\section{Cloning of GsMATE}

Based on the gene expression profiles of Al-resistant Glycine soja (unpublished data), an aluminum-induced gene encoding a citrate transporter of MATE protein was cloned using the sequence of the Glycine soja BW69 line from the NCBI database under accession number BM732932.1. The MATE gene located on soybean chromosome 2 was then designated GsMATE (multidrug and toxin extrusion family protein of Glycine soja). The GSMATE gene was induced in more than twice expression level under the treatment of $25 \mu \mathrm{M} \mathrm{AlCl}_{3}(\mathrm{pH} 4.3$, $0.5 \mathrm{mM} \mathrm{CaCl}_{2}$ ) (data not shown). The full-length genomic GsMATE sequence included 13 exons and 12 introns, with a full-length cDNA of 1,955 bp (data not shown) and open reading frame (ORF) of 1,503 bp encoding 501 amino acids. The GsMATE sequence was deposited in the NCBI database under accession number BM732932.1 (Additional file 2: Table S2).

\section{Bioinformatics analysis of GsMATE}

Phytozome quick search and NCBI BLAST analysis of the conservative domains revealed that GsMATE protein belongs to the MATE family of multidrug and toxin extrusion proteins (Additional file 3: Figure S1a). Prediction using TMHMM (Server v. 2.0) showed that GsMATE protein is a membrane protein with 10 transmembrane helices (domains) at the amino acid positions 145-167, 193-215, 225-247, 254-276, 291-313, 326348, 368-390, 402-424, 434-456, and 463-485. Ring structures were formed using the transmembrane domains (Additional file 3: Figure S1b).

Multiple sequence alignments and homology analysis showed differences among the members of MATE family in plants. As shown in Figs. 1, 42 MATE proteins were clustered into three groups. Group I comprised 17 MATE proteins with diverse potential functions, including regulation of organ initiation (AtZRZ and ZmBIGE1); compound transport and accumulation of alkaloids (NtMATE1), proanthocyanidins (DkMATE1), nicotine (Nt-JAT1, NtMATE1 and NtMATE2), chloride (AtDTX33 and AtDTX35); iron homeostasis (AtBCD); and responses to abiotic stresses such as drought (AtDTX50). Group II comprised only one member, AtEDS5, which is required for salicylic acid (SA) synthesis in pathogen-challenged plants [49]. There were 24 MATE proteins in group III performing major functions in $\mathrm{Al}$-activated citrate transport or the regulation of iron homeostasis in plants (AtFRD3, GmFRD3a, GmFRD3b and OsFRDL1). At the amino acid level, GsMATE showed 56\% similarity with other MATE transporters clustered in Group III, while the similarity among GsMATE, GmMATE13 and GmMATE58 exceeded 95\% (Fig. 1). Therefore, the bioinformatics analysis indicated that GsMATE protein may function in Al tolerance.

\section{GsMATE expression pattern analysis}

To investigate the tissue expression pattern of GsMATE, samples of young root, stem, leaf and cotyledon were taken from the BW69 and JW81 lines of Glycine soja. The analysis of quantitative real-time PCR (qRT-PCR) indicated that GSMATE is expressed constitutively in soybean with more than 3-fold expression levels in roots than those in stems and leaves (Fig. 2a). Further qRT-PCR analysis of GSMATE expression in different segments of the root measured from the tip showed that the highest and lowest expression under the check $\left(0 \mu \mathrm{M} \mathrm{AlCl}_{3}, \mathrm{pH} 4.3,0.5 \mathrm{mM}\right.$ $\mathrm{CaCl}_{2}$ ) in the $0-2 \mathrm{~cm}$ and $4-6 \mathrm{~cm}$ zones, respectively (Fig. 2b). However, the GsMATE gene was significantly 


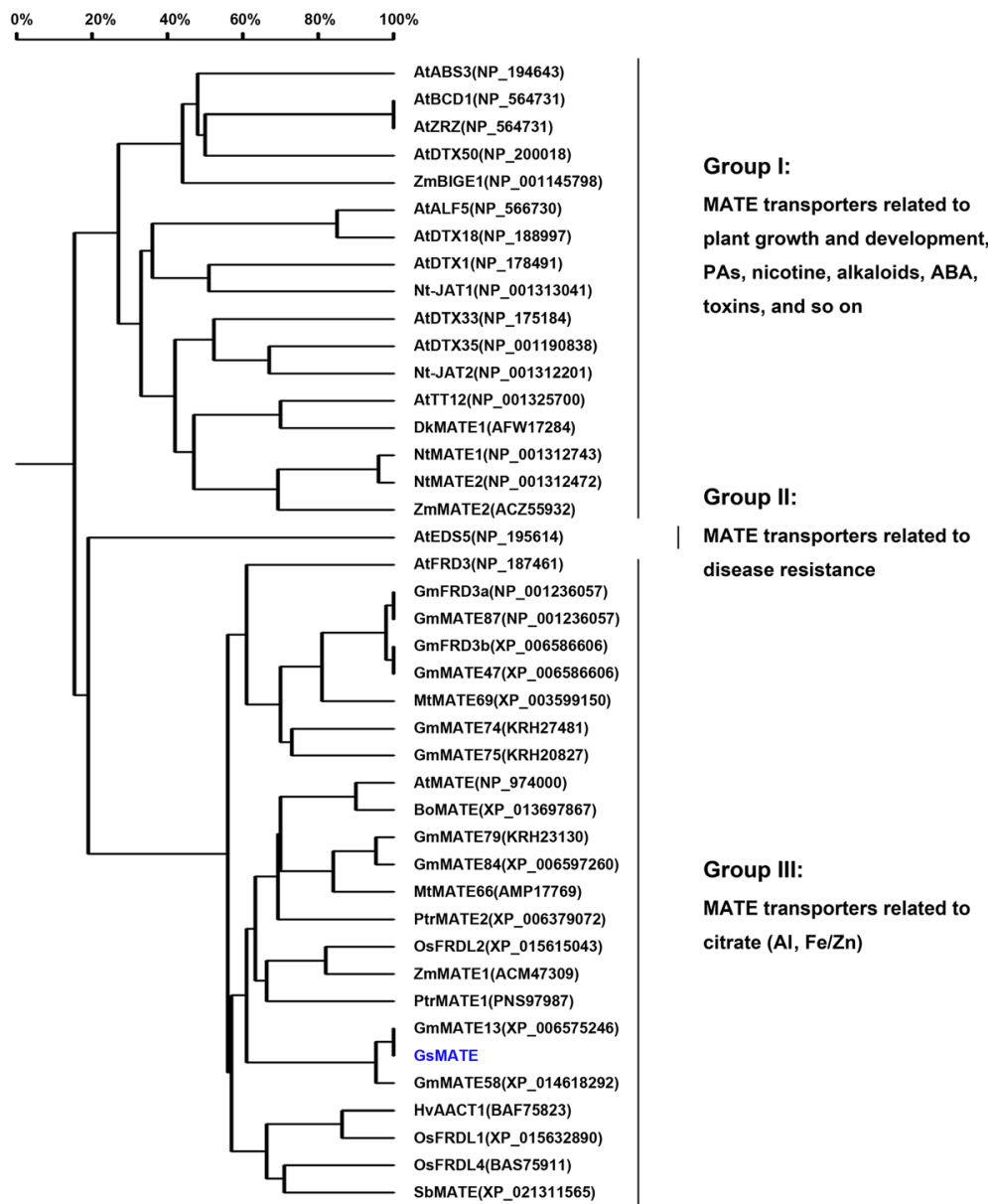

Fig. 1 Homology analysis of GSMATE and other transmembrane proteins. All the available amino acid sequences and the accession numbers of MATE proteins were obtained from the NCBI databases (https://www.ncbi.nlm.nih.gov/). The MATE transporters have been characterized and their functions were identified except for GSMATE and the eight soybean MATE proteins [54]. The homology tree was produced using DNAMAN alignments. The accession numbers of the MATE proteins were shown in parentheses

up-regulated after $\mathrm{Al}$ treatment with almost twice expression levels in the root regions compared with those under the condition of the check (Fig. 2b). The GsMATE expression level at the root segment of the 4-6 cm zone of JW81 line was at least 3-fold expression level than that at the check treatment (Fig. 2b).

\section{Analysis of GsMATE responses to acidic aluminum}

GsMATE was significantly induced under exposure to the different $\mathrm{Al}$ concentration gradients, with 4-fold to 12-fold greater expression levels detected in the BW69 and JW81 wild soybean lines compared with those detected under control conditions (Fig. 3a). The GsMATE gene expressed highest level at the treatment of $50 \mu \mathrm{M} \mathrm{AlCl}$ with a significantly higher level up to 12-fold detected in the aluminum-sensitive JW81 line than those in the aluminum-resistant BW69 line. In contrast, at the higher concentrations of $\mathrm{AlCl}_{3}$ (75 and $\left.100 \mu \mathrm{M}\right)$,
GSMATE expression in the aluminum-resistant BW69 line was significantly higher than that in the aluminum-sensitive JW81 line (Fig. 3a).

The temporal expression pattern of GSMATE in response to acidic aluminum exposure was analyzed over a period of $24 \mathrm{~h}$ (Fig. 3b). Although the expression level of GsMATE was low at $\mathrm{pH}$ 5.8, it was much higher than those detected at $\mathrm{pH} 4.3$ with similar expression patterns observed in both the BW69 and JW81 lines. The Al-resistant BW69 line responded two-hours earlier to $\mathrm{Al}$ stress than the Al-sensitive JW81 line with the highest expression level of GSMATE up to 8.6-fold and 11-fold detected after treatment for $6 \mathrm{~h}$ and $8 \mathrm{~h}$ in the two lines, respectively (Fig. 3b).

\section{Subcellular localization of GsMATE}

To examine its subcellular localization, the GSMATE sequence was fused to the GFP reporter gene at the 5 '-terminus under the control of the CaMV 35S promoter. 


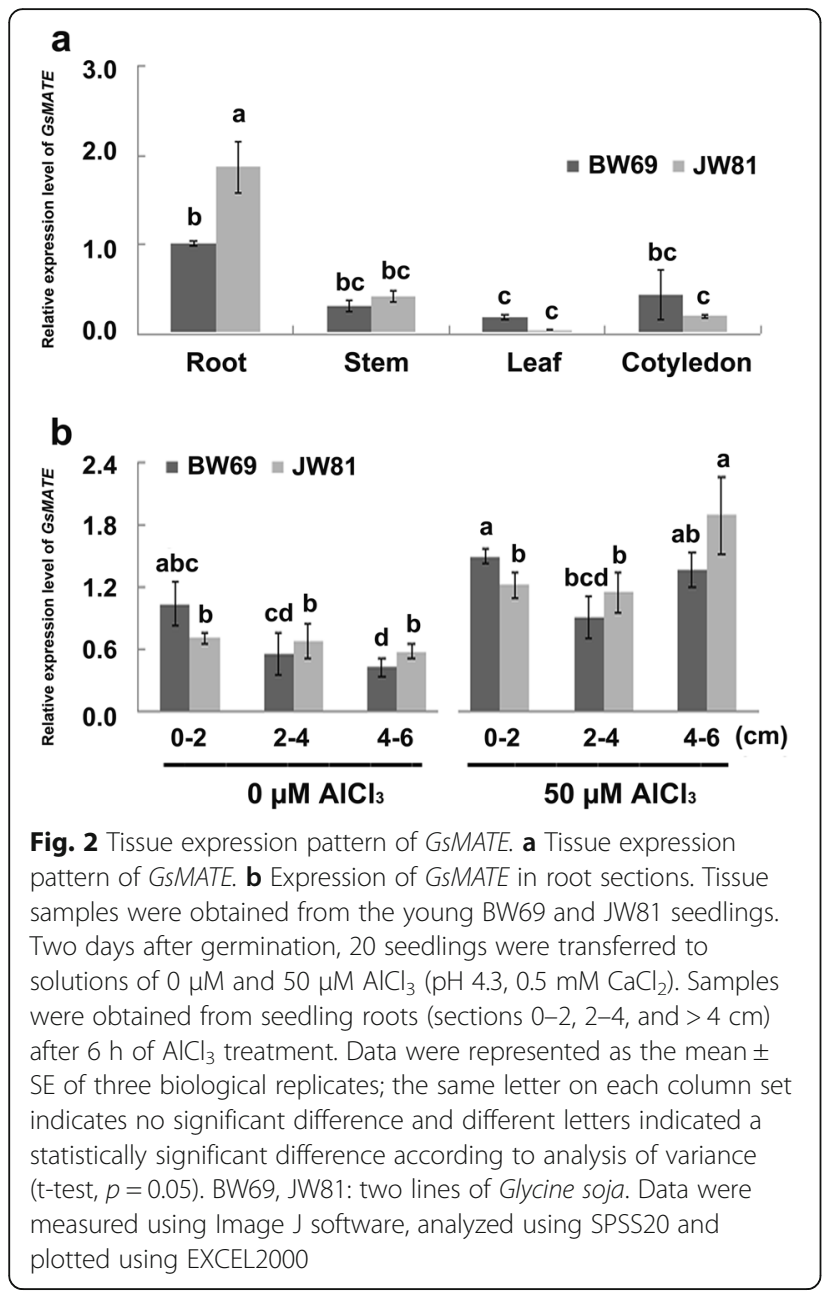

The recombinant constructs encoding the GsMATE-GFP fusion protein and GFP alone (pYL322-d1-eGFP vector) were then transformed into Arabidopsis protoplasts by heat-shock. As shown in Fig. 4a, the GsMATE-GFP fusion protein accumulated mainly in the membrane with strong signals of green fluorescence located in the cell membrane. In contrast, GFP alone was observed as green fluorescence emitted throughout the whole cell (Fig. 4d). These observations were consistent with the predicted function of GsMATE as a membrane protein (Additional file 3: Figure S1b).

\section{Generation and molecular identification of GsMATE transgenic lines}

More than 19 GSMATE transgenic plants of the $\mathrm{T}_{1}$ generation were further identified by PCR amplification of the hygromycin gene encoded by the pCAMBIA1301 vector (data not shown). The results indicated that GSMATE has been integrated into the Arabidopsis thaliana genome (Additional file 4: Figure S2a). Transgenic lines of the $\mathrm{T}_{2}$ generation were identified by qRT-PCR analysis of the GSMATE overexpression in Arabidopsis at the RNA level. Two transgenic GSMATE lines were selected to investigate

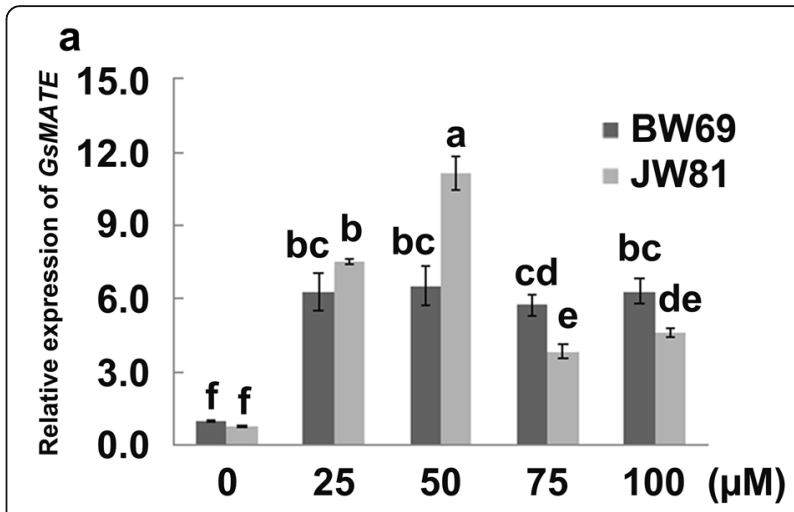

b

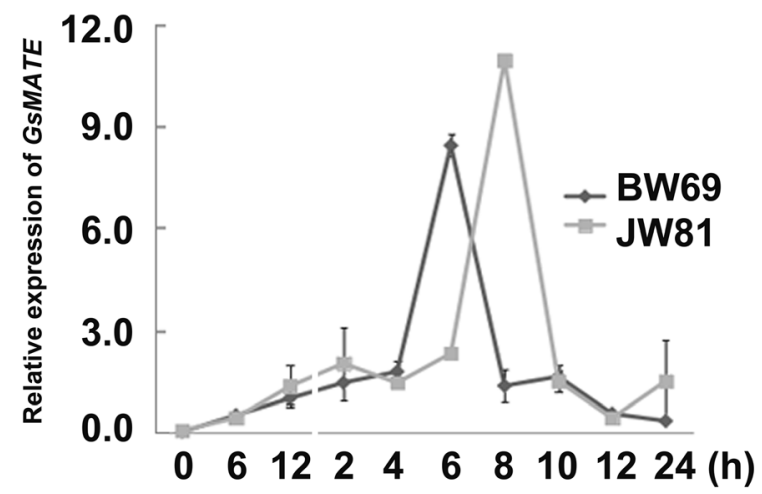

Fig. 3 Pattern of GSMATE expression in response to acidic aluminum exposure. a Pattern of GSMATE expression under acidic aluminum exposure. b Temporal expression pattern of GSMATE under acidic aluminum exposure. Two days after germination, the seedlings were transferred to solutions of $0,25,50,75$, and $100 \mu \mathrm{M} \mathrm{AlCl}$ ( $(\mathrm{pH} 4.3$, $0.5 \mathrm{mM} \mathrm{CaCl}$ ). After $6 \mathrm{~h}$, root tip samples ( $6 \mathrm{~cm}$ long) were obtained from the seedlings for the analysis of the GSMATE expression patterns. To analyze the temporal expression pattern of GSMATE, seedlings (2 days after germination) were cultured in a solution of $0.5 \mathrm{mM} \mathrm{CaCl}_{2}$ ( $\mathrm{pH} 4.3$ ) for $24 \mathrm{~h}$, and then transferred to the solution of $50 \mu \mathrm{M} \mathrm{AlCl}_{3}$ ( $\mathrm{pH} 4.3,0.5 \mathrm{mM} \mathrm{CaCl} 2$ ). Root tip samples $(6 \mathrm{~cm}$ long) were obtained from the seedlings after the treatments of $2,4,6,8,12$, and $24 \mathrm{~h}$. Data were represented as the mean $\pm \mathrm{SE}$ of three biological replicates; the same letter on each column set indicates no significant difference and different letters indicate a statistically significant difference according to analysis of variance (t-test, $p=0.05$ ). BW69, JW81: two lines of Glycine soja. Data were analyzed using SPSS20, and plotted using EXCEL2000

the phenotype and mechanism of resistance to acidic aluminum (Additional file 4: Figure S2b).

\section{GsMATE enhanced the tolerance of Arabidopsis to Al toxicity}

To investigate the responses of GSMATE transgenic lines to $\mathrm{Al}$ stress, Arabidopsis seedlings were exposed to $\mathrm{AlCl}_{3}$ at 0 , 50,100 , and $150 \mu \mathrm{M}$ for 7 days. The results showed that the relative root length (taproots and lateral roots) of GSMATE transgenic lines was significantly greater $(>80 \%)$ than those of the wild-type under $\mathrm{Al}$ stress at 50 and $100 \mu \mathrm{M} \mathrm{AlCl}$ (Fig. 5a). However, under $150 \mu \mathrm{M} \mathrm{AlCl}$, root elongation 


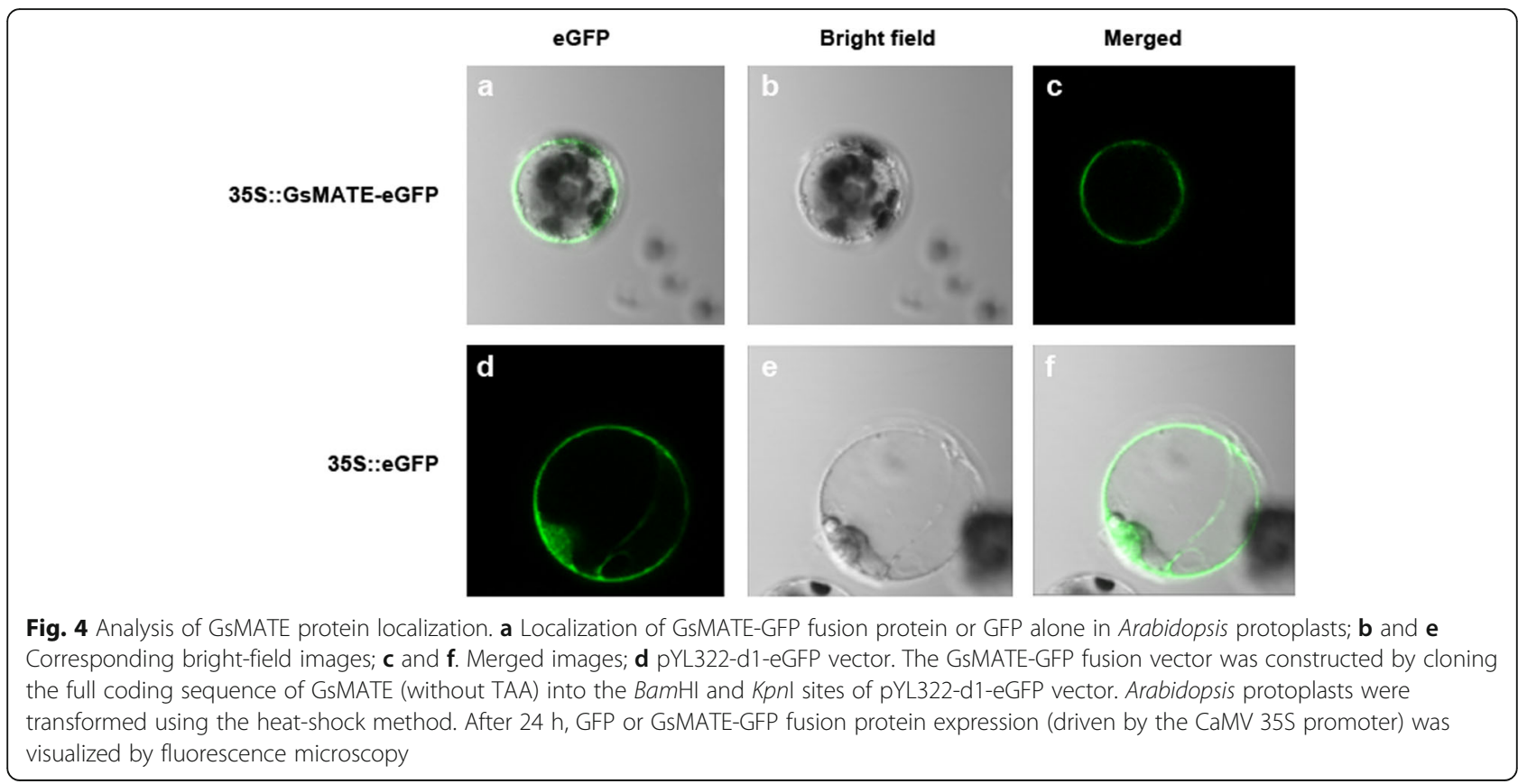

was completely inhibited in both the GsMATE transgenic lines and wild-type Arabidopsis performing similar phenotypes and relative root lengths (Fig. 5a). Furthermore, the Arabidopsis seedlings exhibited similar Al-resistance phenotypes under $\mathrm{Al}$ stress for 2 days (Additional file 5: Figure S3). These results indicated that GSMATE overexpression may improve the tolerance to aluminum stress in Arabidopsis.
To further confirm the involvement of GsMATE in resistance to aluminum toxicity, $\mathrm{T}_{3}$ generation Arabidopsis seedlings were harvested after developing for 12 days in $1 /$ $2 \mathrm{MS}$ media, followed by treatment with $\mathrm{AlCl}_{3}(0,50,100$, and $150 \mu \mathrm{M}$ ) for $30 \mathrm{~min}$. Hematoxylin staining of the roots of GSMATE transgenic lines increased in intensity with the increased concentration of aluminum. Furthermore, at the same concentration of $\mathrm{AlCl}_{3}$, the intensity of
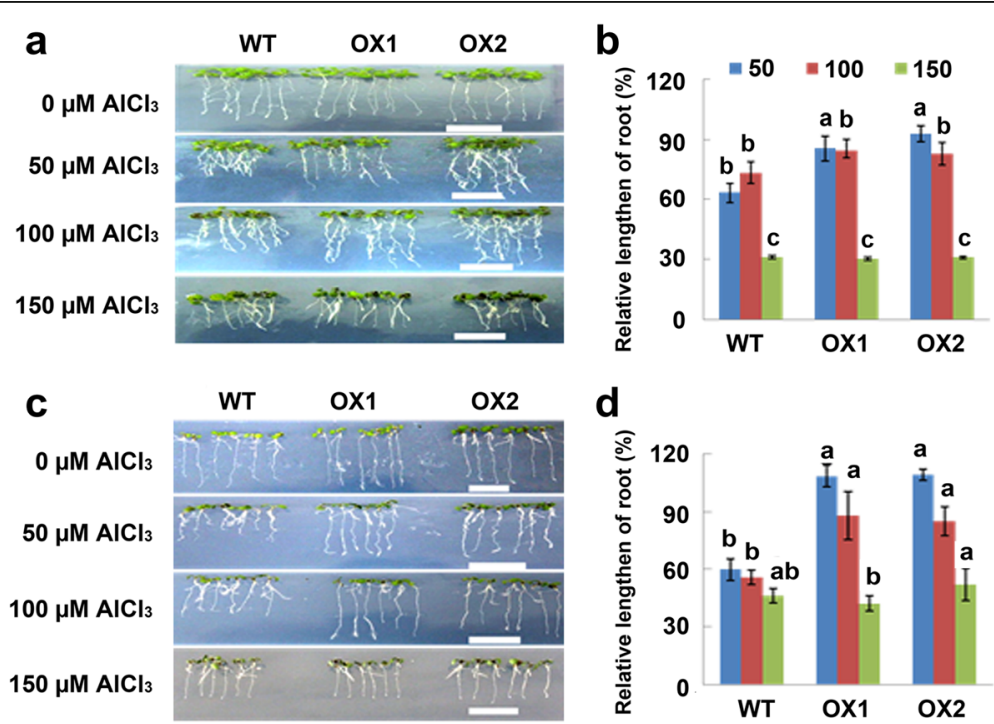

Fig. 5 GSMATE increases Al tolerance of transgenic Arabidopsis lines. a Al tolerance phenotypes of GsMATE in transgenic lines. b Statistical analysis of relative root length. WT: wild-type of Arabidopsis (Col-0); OX1/OX2: GsMATE overexpression transgenic lines. Two days after germination, seedlings were transferred to culture medium containing 0, 50, 100, and $150 \mu \mathrm{M} \mathrm{AlCl} 3(\mathrm{pH} 4.3,0.5 \mathrm{mM} \mathrm{CaCl} 2)$. After 7 days in culture, images of the phenotypes of the GSMATE transgenic lines were recorded for statistical analysis. Data were represented as the mean \pm SE of three biological replicates (t-test, $p=0.05$ ). Data were measured using Image J software, analyzed using SPSS20, and plotted using EXCEL2000 
hematoxylin staining was much greater in the roots of wild-type Arabidopsis seedlings than those of the GsMATE transgenic plants (Additional file 5: Figure S3). These observations indicated that GSMATE may enhance the resistance of transgenic plants to Al toxicity by inhibiting the accumulation of aluminum in the roots.

\section{Discussion}

The present study aimed to investigate the function of GsMATE gene response to acidic aluminum. Previous reports showed that MATE proteins represented a large family in bacteria, fungi, plants and mammals which can transport numerous substrates [14]. The plant MATE proteins characterized to transport citrate are involved in several physiological processes including Al-tolerance [1, 6, 15-19, 21-24, 26], iron translocation [23, 30, 32], heavy metals [33], toxins [34], vacuolar transport of nicotine [3437], chloride channels [38]; ABA efflux [39]; transport of secondary metabolites such as alkaloids, flavonoids, and anthocyanins [36, 40-42]; and phosphorus efficiency. In this study, a GSMATE gene encoding an $\mathrm{Al}$-activated citrate MATE transporter was cloned from the BW69 line of wild soybean and used to characterize the functional properties as well as its potential role in plants. Our results have shown that GsMATE plays a role in citrate secretion. First, GSMATE is expressed mainly in roots with an expression pattern that is specifically up-regulated by $\mathrm{Al}$ with higher expression level in the root tips (Figs. 1 and 2). Second, similar to other MATE transporters involved in Al-induced citrate secretion, the GsMATE protein is also localized predominantly at the plasma membrane in protoplasts (Fig. 4).

The soybean MATE family is large, consisting of at least 117 members located on chromosomes 1 to 20 with uneven distribution. Most MATE genes exhibit tissue-specific expression patterns [54]; however, genome-wide association analysis of MATE transporters showed that soybean MATE family could be classified into four subfamilies comprising a total of ten smaller subgroups with diverse potential functions, including extrusion of compounds, regulation of disease resistance, transport and accumulation of flavonoids or alkaloids, and responses to abiotic stresses [54]. Based on previously reported MATE proteins, further analysis revealed that eight soybean MATE transporters clustered together were related to $\mathrm{Al}$ detoxification and iron translocation [54]. In the present study, the phylogenetic analysis was carried out using the available amino acid sequences of reported MATE proteins and the eight soybean MATE transporters. Homology analysis showed that GsMATE which was clustered with eight Al-induced soybean MATE transporters in Group III covering $\mathrm{Al}$ detoxification and iron translocation (Fig. 1). Amino acid sequence alignment showed that GsMATE has only one different amino acid at the end of C-terminal compared to that of GMMATE47 (data not shown). In addition, GmMATE87 and GmFRD3a have the same sequence, while GMMATE47 has the same sequence as GmFRD3b [31] with the exception of 15 additional amino acids at the end of N-terminal of GmMATE47 (Fig. 1, data not shown). Previous studies showed that the expression of GmFRD3a and GmFRD3b induced by iron deficiency in the iron-efficient reference cultivar Williams 82 played a role in iron translocation in soybean [31]. Differential gene expression analysis indicated that GMMATE47 and GMMATE87 were related to aluminum detoxification and iron translocation. While GmMATE75, which is the candidate gene among the eight identified MATE genes for Al tolerance in soybean, is rapidly up-regulated by $\mathrm{Al}$ stress [54]. In our study, heterogenous overexpression of GSMATE enhanced the resistance to $\mathrm{Al}$ stress in Arabidopsis (Fig. 5). Therefore, our results provided a foundation for further investigation of the functions of soybean MATE genes including the candidate gene for $\mathrm{Al}$ tolerance in soybean.

\section{Conclusions}

The GSMATE gene encodes a transmembrane protein that is enriched in soybean roots, and is up-regulated in response to acidic aluminum. GSMATE overexpression enhances the resistance to $\mathrm{Al}$ toxicity in transgenic Arabidopsis plants. These results indicated that the GsMATE protein may be responsible for external detoxification of $\mathrm{Al}$ by mediating root citrate efflux.

\section{Additional files}

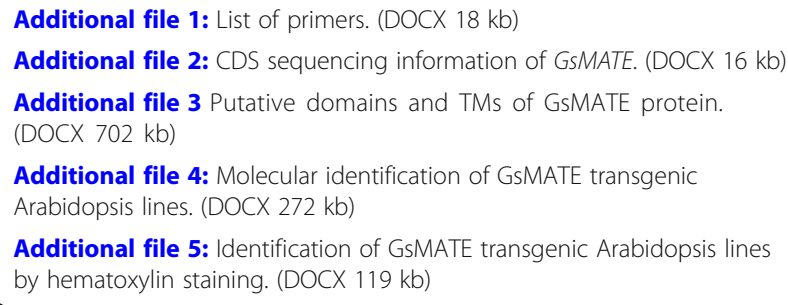

Additional file 4: Molecular identification of GSMATE transgenic Arabidopsis lines. (DOCX 272 kb)

Additional file 5: Identification of GSMATE transgenic Arabidopsis lines by hematoxylin staining. (DOCX $119 \mathrm{~kb}$ )

\section{Abbreviations}

ABA: Abscisic acid; Al: Aluminum; BW69 and JW81: two lines of Glycine soja; CDS: Coding sequence; Gs: Glycine soja; MATE: Multidrug and toxic compound extrusion; NCBI: the National Center for Biotechnology Information; ORF: Open reading frame; OX1/OX2: Overexpression transgenic lines of GSMATE; WT: Wide type of Arabidopsis (Col-0)

\section{Acknowledgements \\ The authors would like to thank Dr. Karen Bysouth (The Peerwith Team) and Dr. Bo Zhang (Assistant Professor, Virginia Tech, USA) for improving the English language of the present manuscript. We are very grateful to Prof. Qinghua Pan and Prof. Yaoguang Liu from South China Agricultural University for kindly providing us with the 35S::ARF19IV-mCherry vector and the pYL322-d1-eGFP vector, respectively.}

\section{Funding}

This work was supported by grants from the National Natural Sciences Foundation of China (31771816, 31371642, 30971814), the Major Projects of New Varieties Cultivation of Genetically Modified Organisms (2016ZX08004002-007), the China Agricultural Research System (CARS-04-PS09), the Research Project of Plant Biology for Undergraduate Students of South China Agricultural University 
(6900-32990369), and the Research Project of the State Key Laboratory for Conservation and Utilization of Subtropical Agro-bioresources (4100-M13024).

\section{Availability of data and materials}

Sequence data are available from Additional file 2 and the NCBI's GenBank under the accession number XM_006575183 (https://blast.ncbi.nlm.nih.gov/ Blast.cgi\#alnHdr_955307808). Any other datasets supporting the conclusions of the present article are contained in text and additional files.

\section{Authors' contributions}

$\mathrm{QM}, \mathrm{XL}$ and $\mathrm{HN}$ designed the research. RY carried out gene cloning, vector construction, expression pattern analysis, genetic transformation of Arabidopsis, and GsMATE protein localization. LL, ZC completed the analysis of the resistance to Al toxicity phenotype. YC and YM reproduced and prepared all the plants for research experiments. $Z \mathrm{~L}, \mathrm{ZT}$, $\mathrm{YZ}$, and $\mathrm{HH}$ carried out the molecular identification of GsMATE transgenic plants. $X Y, X Z$ and $Q Z$ performed the gene sequencing, gene bioinformatics analysis and hematoxylin staining. $\mathrm{QM}, \mathrm{RY}, \mathrm{XL}$ and $\mathrm{HN}$ prepared the manuscript. All authors read and approved the final manuscript.

\section{Ethics approval and consent to participate}

Not applicable.

\section{Consent for publication}

Not applicable.

\section{Competing interests}

The authors declare that they have no competing interests.

\section{Publisher's Note}

Springer Nature remains neutral with regard to jurisdictional claims in published maps and institutional affiliations.

\section{Author details \\ ${ }^{1}$ The State Key Laboratory for Conservation and Utilization of Subtropical Agro- bioresources, South China Agricultural University, Guangzhou, Guangdong 510642, People's Republic of China. ${ }^{2}$ The Key Laboratory of Plant Molecular Breeding of Guangdong Province, College of Agriculture, South China Agricultural University, Guangzhou, Guangdong 510642, People's Republic of China. ${ }^{3}$ The National Engineering Research Center of Plant Space Breeding, South China Agricultural University, Guangzhou, Guangdong 510642, People's Republic of China. ${ }^{4}$ The Experimental Teaching Center of Public Basic Courses, South China Agricultural University, Guangzhou, Guangdong 510642, People's Republic of China. ${ }^{5}$ The Guangdong Provincial Bioengineering Institute, Guangzhou, Guangdong 510316, People's Republic of China. ${ }^{6}$ The Guangdong AIB Polytechnic, Guangzhou, Guangdong 510316, People's Republic of China.}

Received: 25 May 2018 Accepted: 27 August 2018 Published online: 29 September 2018

\section{References}

1. Li N, et al. Genome-wide analysis of MATE transporters and molecular characterization of aluminum resistance in Populus. J Exp Bot. 2017;68(20): 5669-83.

2. Zhang J, et al. The role of the plasma membrane $\mathrm{H}^{+}$-ATPase in plant responses to aluminum toxicity. Front Plant Sci. 2017:8:1757.

3. Kochian LV, et al. Plant adaptation to acid soils: the molecular basis for crop aluminum resistance. Annu Rev Plant Biol. 2015;66:571-98.

4. Kochian LV, Hoekenga OA, Piñeros MA. How do crop plants tolerate acid soils? Mechanisms of aluminum tolerance and phosphorous efficiency. Annu Rev Plant Biol. 2004:55:459-93.

5. Chen $\mathrm{Q}$, et al. Transcriptional and physiological changes of alfalfa in response to aluminium stress. J Agric Sci. 2011;149:737-51.

6. Liu J, et al. Aluminum-activated citrate and malate transporters from the MATE and ALMT families function independently to confer Arabidopsis aluminum tolerance. Plant J. 2009;57(3):389-99.

7. Santos ALD, et al. Global analysis of the MATE gene family of metabolite transporters in tomato. BMC Plant Biol. 2017;17:185.
8. Delhaize $E$, Gruber BD, Ryan PR. The roles of organic anion permeases in aluminium resistance and mineral nutrition. FEBS Lett. 2007;581(12):2255-62.

9. Kinraide TB, Parker DR, Zobel RW. Organic acid secretion as a mechanism of aluminium resistance: a model incorporating the root cortex, epidermis, and the external unstirred layer. J Exp Bot. 2005;56(417):1853-65.

10. Delhaize E, Ryan PR. Aluminum toxicity and tolerance in plants. Plant Physiol. 1995;107:315-21.

11. Delhaize, E., et al., Aluminum tolerance in wheat (Triticum aestivum 1.) (I. Uptake and distribution of aluminum in root apices). 1993. p. 685-693.

12. Kuroda T, Tsuchiya T. Multidrug efflux transporters in the MATE family. Biochimica et Biophysica Acta (BBA) - Proteins and Proteomics. 2009; 1794(5):763-8.

13. He $X$, et al. Structure of a cation-bound multidrug and toxic compound extrusion transporter. Nature. 2010;467(7318):991-4.

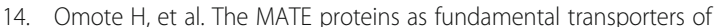
metabolic and xenobiotic organic cations. Trends Pharmacol Sci. 2006; 27(11):587-93.

15. Magalhaes $\mathrm{JV}$, et al. A gene in the multidrug and toxic compound extrusion (MATE) family confers aluminum tolerance in sorghum. Nat Genet. 2007; 39(9):1156-61.

16. Furukawa J, et al. An aluminum-activated citrate transporter in barley. Plant Cell Physiol. 2007;48(8):1081-91.

17. Lei GJ, et al. Two MATE transporters with different subcellular localization are involved in Al tolerance in buckwheat. Plant Cell Physiol. 2017;58(12): 2179-89.

18. Ryan PR, et al. A second mechanism for aluminum resistance in wheat relies on the constitutive efflux of citrate from roots. Plant Physiol. 2009;149(1): $340-51$

19. Maron LG, et al. Two functionally distinct members of the MATE (multi-drug and toxic compound extrusion) family of transporters potentially underlie two major aluminum tolerance QTLs in maize. Plant J. 2010;61(5):728-40.

20. Yokosho K, Yamaji N, Ma JF. An Al-inducible MATE gene is involved in external detoxification of Al in rice. Plant J. 2011;68(6):1061-9.

21. Zhou G, et al. The barley MATE gene, HVAACT1, increases citrate efflux and $\mathrm{Al}^{3+}$ tolerance when expressed in wheat and barley. Ann Bot. 2013;112(3): $603-12$

22. Zhou G, et al. Enhancing the aluminium tolerance of barley by expressing the citrate transporter genes SbMATE and FRD3. J Exp Bot. 2014;65(9):2381-90

23. Wu X, et al. Brassica oleracea MATE encodes a citrate transporter and enhances aluminum tolerance in Arabidopsis thaliana. Plant Cell Physiol. 2014;55(8):1426-36.

24. Yokosho $\mathrm{K}$, et al. Functional analysis of a MATE gene OSFRDL2 revealed its involvement in Al-induced secretion of citrate, but a lower contribution to Al tolerance in rice. Plant Cell Physiol. 2016;57(5):976-85.

25. Ribeiro AP, et al. Overexpression of BdMATE gene improves aluminum tolerance in Setaria viridis. Front Plant Sci. 2017:8:865.

26. Wang J, et al. Diverse functions of multidrug and toxin extrusion (MATE) transporters in citric acid efflux and metal homeostasis in Medicago truncatula. Plant J. 2017;90(1):79-95

27. Yokosho K, Yamaji N, Ma JF. OsFRDL1 expressed in nodes is required for distribution of iron to grains in rice. J Exp Bot. 2016;67(18):5485-94.

28. Xing J, et al. General control nonrepressed protein 5-mediated histone acetylation of ferric reductase defective 3 contributes to iron homeostasis in Arabidopsis. Plant Physiol. 2015;168(4):1309-20.

29. Seo PJ, et al. A Golgi-localized MATE transporter mediates iron homoeostasis under osmotic stress in Arabidopsis. Biochem J. 2012;442(3):551-61.

30. Yokosho $\mathrm{K}$, et al. OsFRDL1 is a citrate transporter required for efficient translocation of iron in rice. Plant Physiol. 2009;149(1):297-305.

31. Rogers $E E$, et al. Two MATE proteins play a role in iron efficiency in soybean J Plant Physiol. 2009:166(13):1453-9.

32. Durrett TP, Gassmann W, Rogers EE. The FRD3-mediated efflux of citrate into the root vasculature is necessary for efficient iron translocation. Plant Physiol. 2007;144(1):197-205.

33. Li $L$, et al. Functional cloning and characterization of a plant efflux carrier for multidrug and heavy metal detoxification. J Biol Chem. 2002;277(7):5360-8.

34. Diener AC, Gaxiola RA, Fink GR. Arabidopsis ALF5, a ultidrug efflux transporter gene family member, confers resistance to toxins. Plant Cell. 2001:13:1625-37.

35. Shitan N, et al. Involvement of the leaf-specific multidrug and toxic compound extrusion (MATE) Ttransporter Nt-JAT2 in vacuolar sequestration of nicotine in Nicotiana tabacum. PLoS One. 2014;9(9):e108789. 
36. Shoji T, et al. Multidrug and toxic compound extrusion-type transporters implicated in vacuolar sequestration of nicotine in tobacco roots. Plant Physiol. 2009;149(2):708-18.

37. Morita M, et al. Vacuolar transport of nicotine is mediated by a multidrug and toxic compound extrusion (MATE) transporter in Nicotiana tabacum. Proc Natl Acad Sci U S A. 2009:106(7):2447-52.

38. Zhang $\mathrm{H}$, et al. Two tonoplast MATE proteins function as turgor-regulating chloride channels in Arabidopsis. Proc Natl Acad Sci. 2017;114(10):E2036-45.

39. Zhang $\mathrm{H}$, et al. A DTX/MATE-type transporter facilitates abscisic acid efflux and modulates ABA sensitivity and drought tolerance in Arabidopsis. Mol Plant. 2014;7(10):1522-32.

40. Yang $\mathrm{S}$, et al. Molecular cloning and functional characterization of DKMATE1 involved in proanthocyanidin precursor transport in persimmon (Diospyros kaki Thunb.) fruit. Plant Physiol Biochem. 2016;108:241-50.

41. Pérez-Díaz $\mathrm{R}$, et al. VVMATE1 and VVMATE2 encode putative proanthocyanidin transporters expressed during berry development in Vitis vinifera L. Plant Cell Rep. 2014;33(7):1147-59.

42. Marinova K, et al. The Arabidopsis MATE transporter TT12 acts as a vacuolar flavonoid/ $/ \mathrm{H}^{+}$-antiporter active in proanthocyanidin-accumulating cells of the seed coat. Plant Cell. 2007;19(6):2023-38.

43. Wang $R$, et al. A subgroup of MATE transporter genes regulates hypocotyl cell elongation in Arabidopsis. J Exp Bot. 2015;66(20):6327-43.

44. Dobritzsch $M$, et al. MATE transporter-dependent export of hydroxycinnamic acid amides. Plant Cell. 2016;28(2):583-96.

45. Burko Y, et al. From organelle to organ: ZRIZI MATE-type transporter is an organelle transporter that enhances organ initiation. Plant Cell Physiol. 2011; 52(3):518-27.

46. Suzuki M, et al. Conserved functions of the MATE transporter BIG EMBRYO in regulation of lateral organ size and initiation rate. Plant Cell. 2015;27(8): 2288-300.

47. Tiwari $\mathrm{M}$, et al. Expression of OSMATE1 and OsMATE2 alters development, stress responses and pathogen susceptibility in Arabidopsis. Sci Rep. 2015;4:3964.

48. Sun $\mathrm{X}$, et al. ADS1 encodes a MATE-transporter that negatively regulates plant disease resistance. New Phytol. 2011;192(2):471-82.

49. Ishihara T, et al. Overexpression of the Arabidopsis thaliana EDS5 gene enhances resistance to viruses. Plant Biol (Stuttg). 2008;10(4):451-61.

50. Wang $L$, et al. The similar and different evolutionary trends of MATE family occurred between rice and Arabidopsis thaliana. BMC Plant Biol. 2016;16:207.

51. Zhu H, et al. Genome wide analysis of MATE-type gene family in maize reveals microsynteny and their expression patterns under aluminum treatment. J Genet. 2016;95(3):691-704.

52. Zhao J, Dixon RA. MATE transporters facilitate vacuolar uptake of epicatechin 3'-O-glucoside for proanthocyanidin biosynthesis in Medicago truncatula and Arabidopsis. Plant Cell. 2009;21(8):2323-40.

53. Lu P, et al. Genome-wide analysis of multidrug and toxic compound extrusion (MATE) family in Gossypium raimondii and Gossypium arboreum and its expression analysis under salt, cadmium, and drought stress. G3 (Bethesda). 2018:2483-500.

54. Liu J, et al. Genome-wide analysis of MATE transporters and expression patterns of a subgroup of MATE genes in response to aluminum toxicity in soybean. BMC Genomics. 2016;17:223.

55. Qiu W, et al. Effects of Fe-deficient conditions on Fe uptake and utilization in P-efficient soybean. Plant Physiol Biochem. 2017;112:1-8.

56. Zeng QY, et al. Identification of wild soybean miRNAs and their target genes responsive to aluminum stress. BMC Plant Biol. 2012;12:182.

57. Lü J, et al. Glyma11g13220, a homolog of the VERNALIZATION pathway gene VERNALIZATION 1 from soybean [Glycine max (L.) Merr.], promotes flowering in Arabidopsis thaliana. BMC Plant Biol. 2015;15:232.

58. Suo $\mathrm{H}$, et al. Overexpression of AtDREB1A causes a severe dwarf phenotype by decreasing endogenous gibberellin levels in soybean Glycine max (L.) Merr. PLoS One. 2012;9(7):e45568.

59. Clough SJ, Bent AF. Floral dip: a simplified method for agrobacteriummediated transformation of Arabidopsis thaliana. The Plant Journal: for cell and molecular biology. 1998;16(6):735

60. Zhang Y, et al. A highly efficient rice green tissue protoplast system for transient gene expression and studying light/chloroplast-related processes. Plant Methods. 2011;7(1):30.
61. Liang $\mathrm{C}$, et al. Low $\mathrm{pH}$, aluminum, and phosphorus coordinately regulate malate exudation through GMALMT1 to improve soybean adaptation to acid soils. Plant Physiol. 2013;161(3):1347-61.

62. Zhu XF, et al. TRICHOME BIREFRINGENCE-LIKE27 affects aluminum sensitivity by modulating the $\mathrm{O}$-acetylation of xyloglucan and aluminum-binding capacity in Arabidopsis. Plant Physiol. 2014;166(1):181-9.

\section{Ready to submit your research? Choose BMC and benefit from:}

- fast, convenient online submission

- thorough peer review by experienced researchers in your field

- rapid publication on acceptance

- support for research data, including large and complex data types

- gold Open Access which fosters wider collaboration and increased citations

- maximum visibility for your research: over $100 \mathrm{M}$ website views per year

At BMC, research is always in progress.

Learn more biomedcentral.com/submissions 